REGARDS

SUR L'ECONOMIE ALLEMANDE

BULLETIN ECONOMIQUE DU CRAC

\section{Regards sur l'économie allemande}

Bulletin économique du CIRAC

$83 \mid 2007$

Varia

\title{
Des risques politiques plutôt que monétaires
}

Isabelle Bourgeois

\section{(2) OpenEdition}

Journals

Édition électronique

URL : http://journals.openedition.org/rea/581

DOI : $10.4000 /$ rea. 581

ISBN : 978-2-8218-0862-1

ISSN : 1965-0787

Éditeur

CIRAC

Édition imprimée

Date de publication : 1 octobre 2007

Pagination : 3-4

ISSN : 1156-8992

Référence électronique

Isabelle Bourgeois, "Des risques politiques plutôt que monétaires », Regards sur l'économie allemande [En ligne], 83 | octobre 2007, mis en ligne le 03 juin 2008, consulté le 15 septembre 2020. URL : http:// journals.openedition.org/rea/581 


\section{Des risques politiques plutôt que monétaires}

Business as usual. C'est ainsi qu'on pourrait résumer l'appréciation de l'évolution de la conjoncture allemande par le monde politique, les milieux économiques et les experts. Certes, les turbulences internationales du crédit inter-bancaire nourrissent une double incertitude sur la croissance américaine et les conditions réservées au financement des entreprises allemandes. Mais un ralentissement de la demande américaine était prévu depuis longtemps; et même s'il s'accélère, ses effets ne seront pas dramatiques pour un commerce extérieur allemand qui a su largement diversifier ses partenaires commerciaux. De même, ce positionnement qui privilégie le marché européen, un espace où l'Allemagne réalise les trois quarts de son excédent commercial, limite les risques inhérents à une dépréciation du dollar d'autant que, dans le même temps, elle compense en partie la flambée des prix pétroliers. Quant à l'impact éventuel de la crise des «subprimes », il ne se laisse pour l'instant pas évaluer. Une chose est certaine : l'indice DAX ne s'est pas effondré, et la solidarité interne des banques allemandes a sauvé de la faillite les banques publiques IKB Industriebank et la Sachsen LB. Et bien que, dans son rapport de septembre, la Bundesbank se refuse à évaluer prématurément les risques toujours possibles d'une restriction du crédit, elle n'en souligne pas moins que la rentabilité des banques allemandes s'est améliorée et que, " globalement, la situation du système bancaire allemand est meilleure que voici quelques années ». Ces incertitudes inquiètent, bien entendu, mais n'entament pas la confiance des acteurs économiques ni des observateurs qui préfèrent concentrer leur attention sur les fondamentaux de l'économie allemande. Or ils sont bons: «les forces de croissance n'ont rien perdu de leur vigueur », affirme ainsi l'IFW de Kiel dans sa note de conjoncture du 20 septembre.

Cela dit, la croissance allemande se ralentit un peu. Mais l'esquisse d'une telle tendance dès le second semestre, fondée sur un léger recul de la demande mondiale, était prévue de longue date. Et si les experts révisent actuellement leurs prévisions 2007 à la baisse, c'est qu'ils corrigent l'euphorie qui s'était emparée d'eux au début de l'été, lorsqu'ils avaient découvert que l'économie allemande "est assise sur des bases beaucoup plus larges qu'on imaginait » (ZEW, voir REA 82/07). Ils avaient alors envisagé pour l'année en cours un taux de croissance plus près de $3 \%$ que des $2,4 \%$ avancés par les Instituts dans leur rapport de printemps. La révision à la baisse n'est donc qu'un « retour à la normale », explique par exemple l'institut HWWI. En revanche, le tassement prévu pour 2008 pourrait être plus important que prévu. Le ministère fédéral de l'Economie maintient pour l'instant son estimation $(+2,4 \%$ ) mais pourrait être amené à la corriger après la parution, fin octobre, du rapport d'automne des instituts de conjoncture. Fait nouveau, ce rapport ne portera pas seulement sur l'analyse et l'évaluation de la conjoncture, mais devra également proposer au gouvernement fédéral des orientations pour son action politique. En outre, sa réalisation est désormais confiée à un groupe de 8 instituts allemands, autrichiens et suisse. Quoi qu'il en soit, la plupart des conjoncturistes envisage pour 2008 et les années suivantes une croissance d'au moins $2 \%$.

Cette confiance se nourrit du fait que, depuis maintenant plusieurs mois, la croissance allemande ne repose plus seulement sur l'export, mais aussi sur l'investissement, à quoi s'ajoute la consommation intérieure. Certes, la hausse du PIB n'a été que de $+0,3 \%$ au second trimestre 2007 par rapport au premier selon Destatis, mais tout de même de $+2,5 \%$ si on compare à 2006. La 'mauvaise performance' s'explique par les effets de la hausse de la TVA et surtout par un recul du BTP, en réalité un retour à la normale après l'hyperactivité enregistrée au cours d'un hiver particulièrement doux. Comme d'habitude, la faiblesse de ce secteur masque les performances de l'industrie : $+5,3 \%$ en un an grâce à des carnets de commande bien remplis.

Cette tendance se poursuit par-delà les variations observées dans les entrées en commande entre juin et juillet, dues à un gonflement exceptionnel en juin liés à des gros contrats conclus dans l'aéronautique. Hors aéronautique, construction navale et ferroviaire, branches où ne se concluent que des affaires hors du commun, le volume des commandes a augmenté de $+2,2 \%$ en juillet dans l'industrie des biens d'investissement, indique le ministère fédéral de l'Economie (rapport de septembre). Ces biens, de même que les biens intermédiaires, connaissent une progression continue depuis le début de l'année, toujours portée par la demande étrangère. Si cette tendance vaut pour l'ensemble de l'industrie (+15,9\% depuis juillet 2006), s'y ajoute désormais la nette hausse des entrées en commande domestiques $(+10,1 \%)$. L'indice de confiance ifo, en se maintenant à un niveau élevé malgré une légère baisse depuis l'été, confirme à sa manière la bonne tenue

Des fondamentaux plus solides qu'il y paraît :...

... export, investissement et consommation

L'industrie au cœur de la croissance 
Emploi en nette hausse et consommation stable

Objectif du gouvernement : "Consolider les bases de la croissance » des activités industrielles, rappelle le ministère. L'institut ifo vient par ailleurs de publier le 27 septembre les résultats d'une enquête menée auprès des industriels dans les secteursphares de l'économie allemande: malgré les charges liées à l'unification et les mutations générées par la globalisation, « l'industrie allemande s'est restructurée avec succès », résume ifo. Si l'export tire ainsi l'acteur-clef de l'économie, sa compétitivité consolidée tire l'investissement en Allemagne : selon Destatis, au second trimestre, les investissements en biens d'équipement ont crû de $+8,8$ \%, secondant ainsi le moteur des exportations (l'excédent commercial a apporté 1,7 point à la croissance au second trimestre). Et ils continueront de progresser nettement, affirme pour sa part l'institut IfW, les entreprises cherchant à bénéficier du régime favorable des amortissements qui prendra fin le 31 décembre.

Tout cela entretient l'embellie sur le marché de l'emploi : le nombre d'actifs occupés a retrouvé en août son niveau d'avant l'unité allemande ; le nombre de chômeurs est tombé à 3,4 millions, soit 700000 de moins en un an, selon l'Agence fédérale pour l'emploi. Mieux encore: selon l'Institut der Deutschen Wirtschaft proche du BDI, le nombre d'emplois vacants s'établit actuellement à 1,5 million, autant qu'en 2000. La dynamique de l'activité et la vitalité du marché de l'emploi soutiennent la consommation privée qui a progressé de $+0,6 \%$ au second trimestre par rapport au premier. En comparaison de mars-mai 2006, elle est restée stable $(-0,2 \%)$, ce qui amène Destatis à se pencher sur sa structure. Les dépenses publiques ayant été en hausse ( $+2,1 \%$ en un an), ce sont bien les ménages qui ont modérément réduit leur consommation après la hausse de la TVA. Comme leurs dépenses de consommation ont globalement crû de $+1,6 \%$ depuis juin 2006 , la retenue observée affecte presque exclusivement l'automobile, les ménages ayant effectué leurs achats avant le 31 décembre. Malgré une hausse de $+1,9 \%$ du revenu disponible, la consommation devrait rester terne, l'inflation atteignant actuellement $2 \%$ (peut-être plus). Quant aux ménages, ils ont reconstitué leur épargne rognée par des achats anticipés: le taux d'épargne est remonté en un an de $10 \%$ à 10,4\%. L'institut GfK, en tout cas, décèle un optimisme foncier chez les consommateurs, lié à la bonne tenue de l'activité.

Tous ces indicateurs donnent raison au ministère fédéral de l'Economie, lorsqu'il affirme dans son rapport de septembre que "l'économie allemande connaît une solide croissance », d'origine structurelle. Cependant, cette robustesse n'est pas acquise une fois pour toutes, surtout dans un environnement mondial de plus en plus compétitif et en permanente évolution. "La prospérité de notre pays doit se conquérir en permanence »: ce constat est au cœur du programme que s'est donné le gouvernement fédéral à Meseberg en août dernier pour la seconde partie de son mandat. " II importe désormais de consolider les bases de la croissance » en poursuivant les réformes, déclare-t-il.

OR CE CAP DE CONSOLIDATION EST ENTACHE D'UN RISQUE POLITIQUE. Certes, la consolidation des finances publiques progresse : cette année, le déficit global de l'Allemagne devrait être ramené à $0,1 \%$. Mieux encore, le budget pourrait être légèrement excédentaire pour la première fois depuis 1989 (hors l'an 2000, exceptionnel du fait de la vente des licences UMTS), Destatis ayant évalué à 1,2 milliard € au premier semestre l'excédent des finances publiques (Bund, Länder, communes et assurance sociale). Du fait de la croissance, les recettes ont en effet été plus importantes que prévu. La réduction du déficit est donc avant tout conjoncturelle, et le ministre fédéral des Finances s'est engagé vis-à-vis de la Commission à réduire de $0,8 \%$ par an le déficit structurel pour le ramener à zéro d'ici 2010. Le gouvernement fédéral s'est fixé le même horizon (« d'ici 2011 ») pour le déficit du Bund. Or de l'avis de la plupart des experts, ces échéances pourraient être rapprochées, la croissance étant désormais bien assise.

Mais en ce début de la deuxième moitié de la législature, la tentation de rompre avec la rigueur, voire d'assouplir le cap des réformes, est grande. Une longue période de campagnes électorales s'ouvre en effet : élections parlementaires dans plusieurs Länder importants en 2008, élections au Bundestag à l'automne 2009. Parallèlement, les deux formations de la grande coalition s'apprêtent à refonder leur programme. Dans ce contexte qui nourrit les exaspérations et les surenchères, le SPD, soucieux de reconquérir sa gauche depuis le recentrage qu'avait fait subir Gerhard Schroeder à son parti avec l'Agenda 2010, se distingue en ranimant une idéologie redistributrice. En atteste son projet de 'correction' des lois Hartz (allongement de la durée de versement des transferts et hausse de l'indemnité forfaitaire ALG II), de même que son soutien actif à l'institution d'un salaire minimum légal. Ces deux revendications sont âprement controversées, et la CDU/CSU partenaire du SPD au sein de la coalition fédérale s'y oppose franchement. Mais si d'aventure, elles parvenaient à s'imposer, elles risqueraient de compromettre le redressement de l'économie allemande - plus sûrement que les risques financiers ou monétaires. Car elles signifieraient un retour en arrière sur l'esprit des réformes en faisant à nouveau prévaloir une logique d'assistance sur une logique de responsabilité. 